\title{
FULLY-DIVERSE LATTICES FROM RAMIFIED CYCLIC EXTENSIONS OF PRIME DEGREE
}

\author{
J. Carmelo Interlando ${ }^{1}$, Antonio A. Andrade ${ }^{2 \S}$, \\ Begoña García Malaxechebarría ${ }^{3}$, \\ Agnaldo J. Ferrari ${ }^{4}$, Robson R. de Araújo ${ }^{5}$ \\ ${ }^{1}$ Department of Mathematics and Statistics \\ San Diego State University \\ San Diego, CA 92182-7720, USA \\ 2 Department of Mathematics \\ São Paulo State University \\ São José do Rio Preto, SP 15054-000, BRAZIL \\ ${ }^{3}$ University of Murcia \\ 30100 Murcia, SPAIN \\ ${ }^{4}$ Department of Mathematics \\ São Paulo State University \\ Bauru, SP 17033-360, BRAZIL \\ ${ }^{5}$ Federal University of São Paulo \\ Cubatão, SP 11533-360, BRAZIL
}

\begin{abstract}
Let $p$ be an odd prime. Algebraic lattices of full diversity in dimension $p$ are obtained from ramified cyclic extensions of degree $p$. The 3,5 , and 7-dimensional lattices are optimal with respect to sphere packing density and therefore are isometric to laminated lattices in those dimensions.
\end{abstract}

AMS Subject Classification: 11H31, 11R18, 11H50, 94B75

Key Words: cyclotomic fields; lattices; modulation diversity; packing density

Received: April 6, 2020

(C) 2020 Academic Publications

${ }^{\S}$ Correspondence author 


\section{Introduction}

In this work lattices mean discrete subgroups of $n$-dimensional Euclidean space. They have been considered in different applied areas, in particular, in coding/modulation theory and more recently in cryptography. They have been studied in several papers from different points of view $[1,3,2,7,11,12]$. In digital communications, two lattice parameters of interest are the sphere packing density and the minimum product distance. This paper presents a construction method of algebraic lattices of optimal packing density and full diversity via totally real number fields of prime degree.

\section{Lattices and number fields}

This section briefly reviews the concepts from lattices and number fields that are required for the rest of the work. Readers interested in further details are referred to [5]. Let $\Lambda$ be a full lattice in $\mathbb{R}^{n}$, that is, $\Lambda$ is the set of all integral linear combinations of some basis of the vector space $\mathbb{R}^{n} . \Lambda$ is said to be of full diversity [3] if for any $\mathbf{0} \neq\left(x_{1}, \ldots, x_{n}\right) \in \Lambda$, one has $x_{i} \neq 0$ for $i=1, \ldots, n$.

Let $\mathfrak{r}$ denote half the minimal distance between (distinct) lattice points. By centering an $n$-dimensional sphere with radius $\mathfrak{r}$ at each lattice point, the sphere packing associated to $\Lambda$ is obtained. The proportion of the space that is occupied by the spheres is called the sphere packing density of $\Lambda$ and is denoted by $\Delta(\Lambda)$. For comparison purposes, a more used parameter is the center density of the packing, denoted by $\delta(\Lambda)$, which in turn equals $\Delta(\Lambda)$ divided by $V_{n}$, the volume of an $n$-dimensional sphere of radius 1 .

Let $\mathbb{K}$ be a number field of degree $n$ and signature $\left[r_{1}, r_{2}\right]$. The $\mathbb{Q}$-monomorphisms (or embeddings) of $\mathbb{K}$ into $\mathbb{C}$ whose images are contained in $\mathbb{R}$ are denoted by $\sigma_{1}, \ldots, \sigma_{r_{1}}$, and those whose images are not contained in $\mathbb{R}$ are denoted by $\sigma_{r_{1}+1}, \overline{\sigma_{r_{1}+1}}, \ldots, \sigma_{r_{1}+r_{2}}, \overline{\sigma_{r_{1}+r_{2}}}$.

Let $\left\{\alpha_{1}, \alpha_{2}, \ldots, \alpha_{n}\right\}$ be any $\mathbb{Z}$-basis for $\mathcal{O}_{\mathbb{K}}$, the ring of integers of $\mathbb{K}$. The integer $d_{\mathbb{K}}=\left(\operatorname{det}\left(\sigma_{j}\left(\alpha_{i}\right)\right)_{i, j=1}^{n}\right)^{2}$ is called the discriminant of $\mathbb{K}$. The trace of any element $x \in \mathcal{O}_{\mathbb{K}}$ is defined by $\operatorname{Tr}_{\mathbb{K} / \mathbb{Q}}(x)=\sum_{i=1}^{n} \sigma_{i}(x)$. For any complex number $z$, let $\mathfrak{R}(z)$ and $\mathfrak{I}(z)$ denote, respectively, its real and imaginary parts. The canonical homomorphism $\sigma: \mathbb{K} \rightarrow \mathbb{R}^{n}$ is defined by

$$
\sigma(x)=\left(\sigma_{1}(x), \ldots, \sigma_{r_{1}}(x), \mathfrak{R}\left(\sigma_{r_{1}+1}(x)\right), \ldots, \mathfrak{I}\left(\sigma_{r_{1}+r_{2}}(x)\right)\right),
$$

for every $x \in \mathbb{K}$. If $\mathcal{M}$ is a $\mathbb{Z}$-submodule of $\mathbb{K}$ of rank $n$, then $\sigma(\mathcal{M})$ is an $n$-dimensional lattice in $\mathbb{R}^{n}$. If either $r_{1}=0$ or $r_{2}=0$, then the center density 
of $\sigma(\mathcal{M})$ is given by

$$
\delta(\sigma(\mathcal{M}))=\frac{t^{n / 2}}{2^{n} \cdot \sqrt{\left|d_{\mathbb{K}}\right|} \cdot\left[\mathcal{O}_{\mathbb{K}}: \mathcal{M}\right]},
$$

where $\left[\mathcal{O}_{\mathbb{K}}: \mathcal{M}\right]$ denotes the index of $\mathcal{M}$ in $\mathcal{O}_{\mathbb{K}}$, and

$$
t=c_{k} \min \left\{\operatorname{Tr}_{\mathbb{K} / \mathbb{Q}}(x \bar{x}): x \in \mathcal{M}, x \neq 0\right\}
$$

with $c_{k}=1$ or $\frac{1}{2}$ according to whether $r_{2}=0$ or $r_{1}=0$, respectively. The quantity $2^{-r_{2}} \cdot \sqrt{\left|d_{\mathbb{K}}\right|} \cdot\left[\mathcal{O}_{\mathbb{K}}: \mathcal{M}\right]$ represents the volume of $\sigma(\mathcal{M})$.

\section{Trace form of cyclic fields of odd prime degree}

This section presents a construction of algebraic lattices using cyclic fields of degree $p$, where $p>2$ is prime and ramified. Let $\mathbb{K} / \mathbb{Q}$ be a cyclic extension of degree $p$. From the Kronecker-Weber Theorem [10], there is a smallest positive integer $n$ such that $\mathbb{K} \subseteq \mathbb{Q}\left(\zeta_{n}\right)$; that integer is the conductor of $\mathbb{K}$. In this case, the discriminant of $\mathbb{K}$ is given by $d_{\mathbb{K}}=n^{p-1}[4, \mathrm{p} .186]$.

Since $p$ is ramified in $\mathbb{K}$, then $n=p^{2} p_{1} p_{2} \cdots p_{r}$ for some $r \geq 0$, where the $p_{i}$ are distinct prime numbers such that $p_{i} \equiv 1(\bmod p)$, see [9], for example. From [8], if $t=\operatorname{Tr}_{\mathbb{Q}\left(\zeta_{n}\right) / \mathbb{K}}\left(\zeta_{n}\right)$, then

1. $\mathbb{K}=\mathbb{Q}(t)$.

2. $\mathcal{B}=\left\{1, \sigma(t), \cdots, \sigma^{p-1}(t)\right\}$ is a $\mathbb{Z}$-basis for $\mathcal{O}_{\mathbb{K}}$.

Theorem 1 ([6]). Let $\mathbb{K}$ be a cyclic field of prime degree $p>2$ and conductor $n$ as above. If $x=a_{0}+\sum_{i=1}^{p-1} a_{i} \sigma^{i}(t) \in \mathcal{O}_{\mathbb{K}}$, where $a_{i} \in \mathbb{Z}$, for $i=0,1, \ldots, p-1$, then

$$
\begin{aligned}
\operatorname{Tr}_{\mathbb{K} / \mathbb{Q}}\left(x^{2}\right) & =p a_{0}^{2}+p p_{1} \cdots p_{r}\left(-2 \sum_{1 \leq i<j \leq p-1} a_{i} a_{j}+(p-1) \sum_{i=1}^{p-1} a_{i}^{2}\right) \\
& =p a_{0}^{2}+p p_{1} \cdots p_{r}\left(\sum_{i=1}^{p-1} a_{i}^{2}+\sum_{1 \leq i<j \leq p-1}\left(a_{i}-a_{j}\right)^{2}\right) .
\end{aligned}
$$




\section{Construction of algebraic lattices}

Let $\mathbb{K} / \mathbb{Q}$ be a cyclic number field of odd prime degree $p$ and conductor $n$ as in Section 3. This section will present a lattice construction whose main ingredient is a suitably chosen $\mathbb{Z}$-submodule $\mathcal{M}$ of $\mathcal{O}_{K}$ of rank $n$. Since $\mathbb{K}$ is totally real, all the obtained lattices will be of full diversity. Their center densities will be calculated by the formula in (1). Recall that the parameter $t$ therein is equal to the nonzero minimum of the trace form of $\mathbb{K}$ restricted to $\mathcal{M}$, see (2).

\subsection{The laminated lattice $\Lambda_{3}$}

In this section, let $\mathbb{K}$ be the cyclic field of degree $p=3$ and conductor $n=3^{2}$. The Galois group $\operatorname{Gal}(\mathbb{K} / \mathbb{Q})=\langle\sigma\rangle$ is cyclic of order $3, t=\operatorname{Tr}_{\mathbb{Q}\left(\zeta_{3^{2}}\right) / \mathbb{K}}\left(\zeta_{3^{2}}\right)$, and $d_{\mathbb{K}}=3^{4}$. Let $\mathcal{M}$ be the submodule of $\mathcal{O}_{\mathbb{K}}$ of rank 3 and index 6 given by

$$
\begin{aligned}
\mathcal{M}= & \left\{a_{0}+a_{1} \sigma(t)+a_{2} \sigma^{2}(t) \in \mathcal{O}_{\mathbb{K}}: a_{0}, a_{1}, a_{2} \in \mathbb{Z}\right. \text { and } \\
& \left.a_{0}+2 a_{1}+2 a_{2} \equiv 0(\bmod 6)\right\} .
\end{aligned}
$$

If $\alpha \in \mathbb{K}$, then

$$
\operatorname{Tr}_{\mathbb{K} / \mathbb{Q}}\left(\alpha^{2}\right)=3 a_{0}^{2}+6 a_{1}^{2}-6 a_{1} a_{2}+6 a_{2}^{2} .
$$

It follows that $\min \left\{\operatorname{Tr}_{\mathbb{K} / \mathbb{Q}}\left(\alpha^{2}\right): \alpha \in \mathcal{M}, \alpha \neq 0\right\}=18$ is attained when $\left(a_{0}, a_{1}, a_{2}\right)=(2,-1,0)$. Since the volume of $\sigma(\mathcal{M})$ equals $\sqrt{\left|d_{\mathbb{K}}\right|} \cdot\left[\mathcal{M}: \mathcal{O}_{\mathbb{K}}\right]=$ $3^{2} \cdot 6=54$, one has

$$
\delta(\sigma(\mathcal{M}))=\frac{(\sqrt{18} / 2)^{3}}{54}=\frac{1}{4 \sqrt{2}},
$$

i.e., the center density of $\sigma(\mathcal{M})$ equals that of lattice $\Lambda_{3}[5$, p. 15].

\subsection{The laminated lattice $\Lambda_{5}$}

In this section, let $\mathbb{K}$ be the number field of degree $p=5$ and conductor $n=5^{2}$. The Galois group $\operatorname{Gal}(\mathbb{K} / \mathbb{Q})=\langle\sigma\rangle$ is cyclic of order $5, t=\operatorname{Tr}_{\mathbb{Q}\left(\zeta_{5^{2}}\right) / \mathbb{K}}\left(\zeta_{5^{2}}\right)$, and $d_{\mathbb{K}}=5^{8}$. Let $\mathcal{M}$ be the submodule of $\mathcal{O}_{\mathbb{K}}$ of rank 5 and index 10 given by

$$
\begin{aligned}
\mathcal{M}=\{ & \left\{a_{0}+a_{1} \sigma(t)+a_{2} \sigma^{2}(t)+a_{3} \sigma^{3}(t)+a_{4} \sigma^{4}(t) \in \mathcal{O}_{\mathbb{K}}: a_{0}, \ldots,\right. \\
& \left.a_{4} \in \mathbb{Z} \text { and } a_{0}+4 a_{1}+4 a_{2}+4 a_{3}+4 a_{4} \equiv 0 \quad(\bmod 10)\right\} .
\end{aligned}
$$

If $\alpha \in \mathbb{K}$, then

$$
\begin{aligned}
\operatorname{Tr}_{\mathbb{K} / \mathbb{Q}}\left(\alpha^{2}\right)= & 5 \cdot\left(a_{0}^{2}+4 a_{1}^{2}-2 a_{1} a_{2}-2 a_{1} a_{3}-2 a_{1} a_{4}+4 a_{2}^{2}\right. \\
& \left.-2 a_{2} a_{3}-2 a_{2} a_{4}+4 a_{3}^{2}-2 a_{3} a_{4}+4 a_{4}^{2}\right) .
\end{aligned}
$$


It follows that $\min \left\{\operatorname{Tr}_{\mathbb{K} / \mathbb{Q}}\left(\alpha^{2}\right): \alpha \in \mathcal{M}\right\}=50$ is attained when $a_{0}=a_{1}=a_{2}=$ $0, a_{3}=-1$ and $a_{4}=1$. Since the volume of $\sigma(\mathcal{M})$ equals $\sqrt{\left|d_{\mathbb{K}}\right|} \cdot\left[\mathcal{M}: \mathcal{O}_{\mathbb{K}}\right]=$ $5^{4} \cdot 10=5^{5} \cdot 2$, one has

$$
\delta(\sigma(\mathcal{M}))=\frac{(\sqrt{50} / 2)^{5}}{5^{5} \cdot 2}=\frac{1}{8 \sqrt{2}},
$$

i.e., the center density of $\sigma(\mathcal{M})$ equals that of lattice $\Lambda_{5}[5$, p.15].

\subsection{The laminated lattice $\Lambda_{7}$}

In this section, let $\mathbb{K}$ be the number field of degree $p=7$ and conductor $n=7^{2}$. The Galois group $\operatorname{Gal}(\mathbb{K} / \mathbb{Q})=\langle\sigma\rangle$ is cyclic of order $7, t=\operatorname{Tr}_{\mathbb{Q}\left(\zeta_{7^{2}}\right) / \mathbb{K}}\left(\zeta_{7^{2}}\right)$, and $d_{\mathbb{K}}=7^{12}$. Let $\mathcal{M}$ be the submodule of $\mathcal{O}_{\mathbb{K}}$ of rank 7 and index 112 given by

$$
\mathcal{M}=\left\{\begin{array}{l}
a_{0}+a_{1} \sigma(t)+a_{2} \sigma^{2}(t)+\cdots+a_{6} \sigma^{6}(t) \in \mathcal{O}_{\mathbb{K}}: \\
a_{0}, a_{1}, a_{2}, \ldots, a_{6} \in \mathbb{Z} \\
a_{0}+6 a_{1}+6 a_{2}+6 a_{3}+6 a_{4}+6 a_{5}+6 a_{6} \equiv 0 \quad(\bmod 14) \\
a_{1}+a_{5}+a_{6} \equiv 0 \quad(\bmod 2), \\
a_{2}+a_{4}+a_{6} \equiv 0 \quad(\bmod 2), \text { and } \\
a_{3}+a_{4}+a_{5}+a_{6} \equiv 0 \quad(\bmod 2)
\end{array}\right.
$$

If $\alpha \in \mathbb{K}$, then

$$
\begin{aligned}
\operatorname{Tr}_{\mathbb{K} / \mathbb{Q}}\left(\alpha^{2}\right)= & 7 \cdot\left(a_{0}^{2}+6 a_{1}^{2}-2 a_{1} a_{2}-2 a_{1} a_{3}-2 a_{1} a_{4}-2 a_{1} a_{5}\right. \\
& -2 a_{1} a_{6}+6 a_{2}^{2}-2 a_{2} a_{3}-2 a_{2} a_{4}-2 a_{2} a_{5}-2 a_{2} a_{6} \\
& +6 a_{3}^{2}-2 a_{3} a_{4}-2 a_{3} a_{5}-2 a_{3} a_{6}+6 a_{4}^{2}-2 a_{4} a_{5} \\
& \left.-2 a_{4} a_{6}+6 a_{5}^{2}-2 a_{5} a_{6}+6 a_{6}^{2}\right) .
\end{aligned}
$$

It follows that $\min \left\{\operatorname{Tr}_{\mathbb{K} / \mathbb{Q}}\left(\alpha^{2}\right): \alpha \in \mathcal{M}\right\}=196$ is attained when $a_{0}=a_{6}=2$ and $a_{1}=a_{2}=a_{3}=a_{4}=a_{5}=0$. Since the volume of $\sigma(\mathcal{M})$ equals $\sqrt{\left|d_{\mathbb{K}}\right|} \cdot[\mathcal{M}$ : $\left.\mathcal{O}_{\mathbb{K}}\right]=7^{6} \cdot 112=2^{4} \cdot 7^{7}$, one has

$$
\delta(\sigma(\mathcal{M}))=\frac{(\sqrt{196} / 2)^{7}}{2^{4} \cdot 7^{7}}=\frac{1}{16},
$$

i.e., the center density of $\sigma(\mathcal{M})$ equals that of lattice $\Lambda_{7}[5$, p. 15]. 


\section{Conclusion}

A method for constructing laminated lattices $\Lambda_{p}$ from cyclic fields of odd prime degree $p$ and conductor $p^{2}$, where $p$ is ramified, was presented. Explicit numerical examples were given for $p=3,5$, and 7. All the obtained lattices have maximal diversity; however, the determination of their exact minimum product distances is left for future research.

\section{Acknowledgment}

This work was supported by CNPq (Conselho Nacional de Desenvolvimento Científico e Tecnológico), Brazil, 429346/2018-2 and FAPESP (São Paulo Research Foundation) 2013/25977-7.

\section{References}

[1] A. A. Andrade, C. Alves and T. B. Carlos, Rotated lattices via the cyclotomic field $\mathbb{Q}\left(\zeta_{2^{r}}\right)$, Int. J. Appl. Math., 19 (2006), 321-331.

[2] A. A. Andrade and J. C. Interlando, Construction of even-dimensional lattices of full diversity, Int. J. Appl. Math., 32, No 2 (2019), 325-332; doi: 10.12732/ijam.v32i2.12.

[3] E. Bayer-Fluckiger, F. Oggier, and E. Viterbo, Algebraic lattice constellations: Bounds on performance, IEEE Trans. Inform. Theory, 52 (2006), 319-327.

[4] P. E. Conner and R. Perlis, A Survey of Trace Forms of Algebraic Number Fields, World Scientific Publishing Co Pte Ltd., Singapore (1984).

[5] J. H. Conway, N. J. A. Sloane, Sphere Packings, Lattices, and Groups, 3rd Ed., Springer-Verlag, New York (1999).

[6] R. R. de Araujo, A. C. M. M. Chagas, A. A. Andrade and T. P. Nóbrega Neto, Trace form associated to cyclic number fields of ramified odd prime degree, J. Algebra App., (2019), Art. 2050080.

[7] J. C. Interlando, J. O. D. Lopes and T. P. N. Neto, A new number field construction of the $D_{4}$-lattice, Int. J. Appl. Math., 31, No 2 (2018), 299305; doi: 10.12732/ijam.v31i2.11. 
[8] G. Lettl, The ring of integers of an Abelian number field, J. Reine Angew. Math., 404 (1990), 162-170.

[9] B. K. Spearman and K. S. Wiliams, The discriminant of a cyclic field of odd prime degree, Rocky Mountain J. Math, 33 (2003), 1001-1122.

[10] L. C. Washington, Introduction to Cyclotomic Fields, 2nd Ed., SpringerVerlag, New York (1997).

[11] C. C. T. Watanabe, J. C. Belfiore, E. D. Carvalho and J. V. Filho, $E_{8^{-}}$ lattice via the cyclotomic field $\mathbb{Q}\left(\zeta_{24}\right)$, Int. J. Appl. Math., 31, No 1 (2018), 63-71; doi: 10.12732/ijam.v31i1.6.

[12] C. C. T. Watanabe, J. C. Belfiore, E. D. Carvalho and J. V. Filho, Construction of nested real ideal lattices for interference channel coding, Int. J. Appl. Math., 32, No 2 (2019), 295-323; doi: 10.12732/ijam.v32i2.11. 
